\title{
Predictive Value of Neutrophil-to-Lymphocyte, Aspartate-to-Alanine Aminotransferase, Lymphocyte-to-Monocyte and Platelet-to-Lymphocyte Ratios in Severity and Side of Carotid Artery Stenosis: Are Those Significant?
}

\author{
Özgür Altinbas, ${ }^{1}$ Şeniz Demiryurek, ${ }^{2}$ Mehmet Isik, ${ }^{3}$ Ömer Tanyeli, ${ }^{3}$ Yüksel Dereli, ${ }^{3}$ Niyazi Gormus ${ }^{3}$ \\ ${ }^{1}$ Gaziantep University, Vocational School of Health Services, Department of Operating Room Services, Gaziantep, Turkey; \\ ${ }^{2}$ Gaziantep University, Medical Faculty, Department of Physiology, Gaziantep, Turkey; ${ }^{3}$ Necmettin Erbakan University, \\ Medical Faculty, Department of Cardiovascular Surgery, Konya, Turkey
}

\section{ABSTRACT}

Background: Atherosclerosis is a chronic disease that leads to mortality and morbidity by affecting arterial vascular structures. Carotid artery is one of these arterial structures and occlusive disease of carotid artery may cause stroke or cranial ischemic infarction. Inflammation plays a role in the atherosclerotic process. In this study, we aimed to discuss the relationship between the severity and side of carotid artery occlusion and novel inflammatory parameters include platelet-to-lymphocyte, neutrophil-tolymphocyte, lymphocyte-to-monocyte, and aspartate-toalanine aminotransferase ratios.

Methods: One-hundred-fifteen patients who had carotid artery stenosis between 50\%-99\% and 115 healthy subjects with no carotid artery stenosis or additional disease were included in the study. The relationship between the side and degree of the lesion and platelet-to-lymphocyte, neutrophilto-lymphocyte, lymphocyte-to-monocyte, and aspartate-toalanine aminotransferase ratios were studied in the patient group. The patients with carotid artery stenosis and the healthy subjects were compared, in the terms of same parameters. Data were evaluated statistically.

Results: There were no statistically significant differences between the groups, in the terms of platelet-to-lymphocyte, neutrophil-to-lymphocyte, lymphocyte-to-monocyte, and aspartate-to-alanine aminotransferase ratios and the degree of stenosis. There was no statistically significant difference between the sides of the lesions and the parameters above except lymphocyte-to-monocyte ratio. It was statistically significantly higher in left-sided lesions. Aspartate-toalanine aminotransferase and neutrophil-to-lymphocyte ratios were markedly higher in the patient group, when compared to controls.

Conclusion: Platelet-to-lymphocyte, neutrophil-tolymphocyte, lymphocyte-to-monocyte, and aspartate-toalanine aminotransferase ratios are inexpensive, easy, fast, and

Received October 27, 2020; accepted November 16, 2020.

Correspondence: Özgür Altinbas, Asst. Prof. Dr., Gaziantep University, Vocational School of Health Services, Department of Operating Room Services, Gaziantep, Turkey;+90-505-657-05-77 (e-mail: ozgur_altinbas@yaboo.com). reproducible parameters that can be used in determining the prediction of carotid artery stenosis.

\section{INTRODUCTION}

Carotid artery stenosis (CAS) is one of the arterial occlusive diseases that may enable mortality or morbidity, due to cranial ischemic infarction and stroke. Atherosclerosis plays a role in $90 \%$ of the cases [Varim 2016]. Atherosclerosis is a chronic and systemic inflammatory disease and affects the arterial intima [Tsiara 2003]. Inflammation is involved in every stage of its process [Kristensen 1992].

Several markers related with inflammation include C-reactive protein, white blood cells, interleukin-6, tumor necrosis factor alpha, amyloid-a, homocysteine and fibrinogen; this is available in the literature [Chrysohoou 2004]. In addition to these indicators, platelet-to-lymphocyte (Plt/ Lym), neutrophil-to-lymphocyte (Neut/Lym), lymphocyteto-monocyte (Lym/Mono), and aspartate-to-alanine aminotransferase (AST/ALT) ratios are recently introduced biochemical markers for inflammation-associated diseases [Gasparyan 2019; Zhang 2020; Kim 2020; Yayla 2016].

Our study is unique, in terms of evaluation of these four novel parameters in one study about carotid artery disease. We aimed to discuss the relationship between the severity and side of carotid artery occlusion and Plt/Lym, Neut/Lym, Lym/Mono, and AST/ALT ratios.

\section{MATERIALS AND METHODS}

Between January 2014 and January 2019, 115 patients who underwent carotid magnetic resonance angiography (MRA) and who had stenosis over $50 \%$ and under $99 \%$ in the right, left, or bilateral carotid arteries and 115 healthy subjects with no CAS or additional diseases were included in this study. Written informed consent was taken from all participants, and the Clinical Ethics Committee of the institution approved this retrospective study protocol. Clinical data about the accompanied disease, such as hypertension (HT), cerebrovascular disease (CVD), diabetes mellitus (DM), coronary artery disease (CAD), 
Table 1. Comparison of demographical and biochemical parameters between patients with CAS and control group.

\begin{tabular}{|c|c|c|c|}
\hline Age (years)* & $66.34 \pm 12.53$ & $68.21 \pm 8.19$ & .1817 \\
\hline Male $(n, \%)$ & $77(67.0)$ & $83(72.2)$ & \\
\hline Female $(\mathrm{n}, \%)$ & $38(33.0)$ & $32(27.8)$ & .4737 \\
\hline MPV (fl) & $9.85 \pm 1.28$ & $9.60 \pm 1.68$ & .2056 \\
\hline РCT (\%) & $0.25 \pm 0.06$ & $0.25 \pm 0.08$ & .9999 \\
\hline Lymphocytes $\left(\times 10^{3} / \mathrm{mm}^{3}\right)$ & $2.32 \pm 0.61$ & $2.40 \pm 0.79$ & .3909 \\
\hline Monocytes $\left(\times 10^{3} / \mathrm{mm}^{3}\right)$ & $0.54 \pm 0.15$ & $0.66 \pm 0.25$ & $<.0001$ \\
\hline Neutrophils $\left(\times 10^{3} / \mathrm{mm}^{3}\right)$ & $4.15 \pm 1.17$ & $5.35 \pm 1.42$ & $<.0001$ \\
\hline Neutrophils/Lymphocytes ratio & $1.86 \pm 0.62$ & $2.45 \pm 1.03$ & $<.0001$ \\
\hline Lymphocytes/Monocytes ratio & $4.34 \pm 1.09$ & $4.07 \pm 2.36$ & .2665 \\
\hline AST/ALT ratio & $0.97 \pm 0.26$ & $1.16 \pm 0.42$ & $<.0001$ \\
\hline \multicolumn{4}{|l|}{ Comorbidity (n, \%) } \\
\hline Hypertension & - & $54(46.9 \%)$ & \\
\hline Cerebrovascular Disease & - & $15(13 \%)$ & \\
\hline Diabetes Mellitus & - & $16(13.9 \%)$ & \\
\hline Coronary Artery Disease & - & $25(21.7 \%)$ & \\
\hline Peripheral Artery Disease & - & $12(10.4 \%)$ & \\
\hline
\end{tabular}

*Data are mean \pm SD. MPV, mean platelet volume; AST, aspartate aminotransferase; ALT, alanine aminotransferase; PCT, plateletcrit. PCT is calculated according to the following formula [Temel 2017]: PCT = platelet count $\times$ MPV $/ 10000$.

peripheral arterial disease (PAD), and chronic obstructive pulmonary disease (COPD) were recorded. Demographic data of the participants, such as gender and age, also were noted. All patients underwent carotid Doppler ultrasound before MRA.

The degree of stenosis was evaluated, according to the North American Symptomatic Carotid Endarterectomy Trial (NASCET) 2011 ASA/ACCF/AHA/AANN/AANS/ACR/ ASNR/CNS/SAIP/SCA Guideline on the Management of Patients with Extracranial Carotid and Vertebral Artery Disease Criteria [Brott 2011].

Initially, groups were divided into two: control group and patient group. Patients were divided into three subgroups, according to their degree of stenosis. Group 1 had a stenosis between $50 \%$ to $69 \%$, Group 2 had a stenosis between $70 \%$ to $89 \%$, and Group 3 had a stenosis between $90 \%$ to $99 \%$.

Side of the lesion was also divided into three groups. Side 1 was right-sided, Side 2 was left-sided, and Side 3 was both-sided.
Data from all patients were collected from hospital medical records. Exclusion criteria were hematologic disease, active infection, simultaneous carotid and valve surgery with coronary artery bypass graft operation, chronic inflammatory or autoimmune diseases and any diagnosed cancer.

Venous blood samples were obtained from patients for hemogram and biochemical analysis. Complete blood cell counts, and automated differential counts were determined via an automated hematology analyzer (Cell-Dyn 3700, Abbott, Germany), which provided total white blood cell, platelet, neutrophil, lymphocyte, monocyte, eosinophil, and basophils counts/ml. The baseline Plt/Lym, Neut/Lym and $\mathrm{Lym} /$ Mono ratios were calculated by dividing the absolute counts of these parameters. Aspartate and alanine aminotransferases were determined via Cobas 8000 (Roche, Germany), and AST/ALT ratio was calculated by dividing the absolute counts of these parameters.

Statistical analysis: Data were expressed as mean \pm standard deviation (SD) or percentage. Statistical analysis was performed using GraphPad Instat version 3.05 (GraphPad 

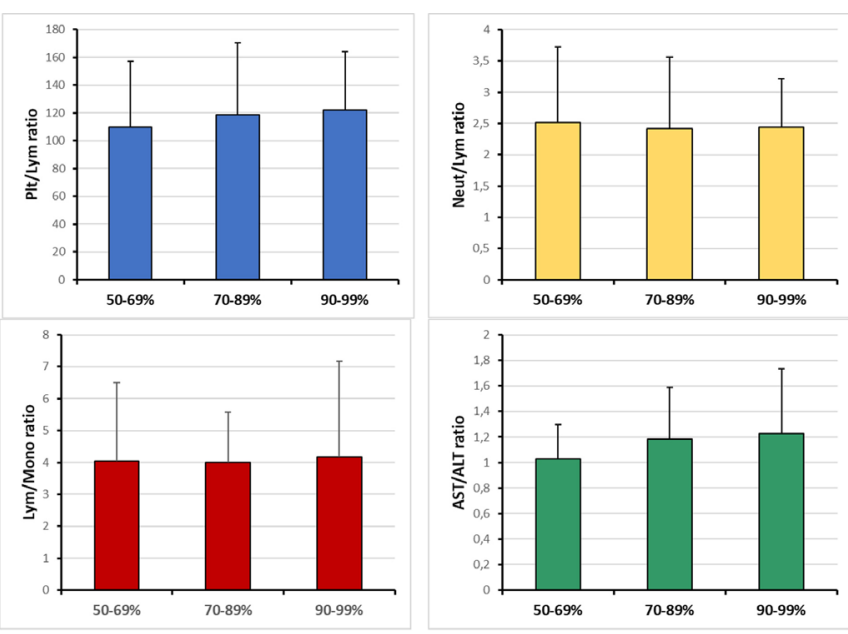

Figure 1. Comparison of Plt/Lym, Neut/Lym, Lym/Mono and AST/ ALT ratios and degree of CAS.

Software Inc., San Diego, CA, USA). For comparisons of the differences between mean values of two groups, the unpaired Student's t test was used. For comparisons of the differences between mean values of three groups, the one-way ANOVA test followed by Student-Newman-Keuls test was used. The Chi-square test was used for calculation of the significance of differences in gender. The Pearson's test was used to identify the correlations. All statistical tests and $P$ values were twosided, and $P<.05$ was considered statistically significant.

\section{RESULTS}

One hundred fifteen patients with CAS and 115 healthy subjects were included in this study. There were no marked differences in age, gender, platelet count, MPV, PCT, lymphocyte count, and ALT between the groups (Table 1).

Initially, patients were divided into three groups, according to the degree of stenosis. There were no statistically significant differences between the groups, in the terms of Plt/ Lym, Neut/Lym, Lym/Mono, and AST/ALT ratios and the degree of stenosis $(P>.05$ for all) (Figure 1).

Similarly, side of the stenosis was divided into three groups and compared in the terms of Plt/Lym, Neut/Lym, Lym/Mono, and AST/ALT ratios (Figure 2). According to the results, there was no statistically significant difference except Lym/Mono ratio. Lym/Mono ratio was statistically significantly higher in left-sided lesions than right-sided lesions $(P<.05)$.

Afterward, patient and control groups were compared in the subject of demographical and biochemical parameters as shown in Table 1. We observed that monocyte, neutrophil counts, and AST value were also significantly higher in the patient group $(P<.0001)$. We also detected that AST/ALT, and Neut/Lym ratios were markedly higher in the patient group, when compared with controls $(P<.0001)$.

Correlations between ratios and biochemical parameters in patients with CAS also were compared and results were given in Table 2. There were positive correlations between
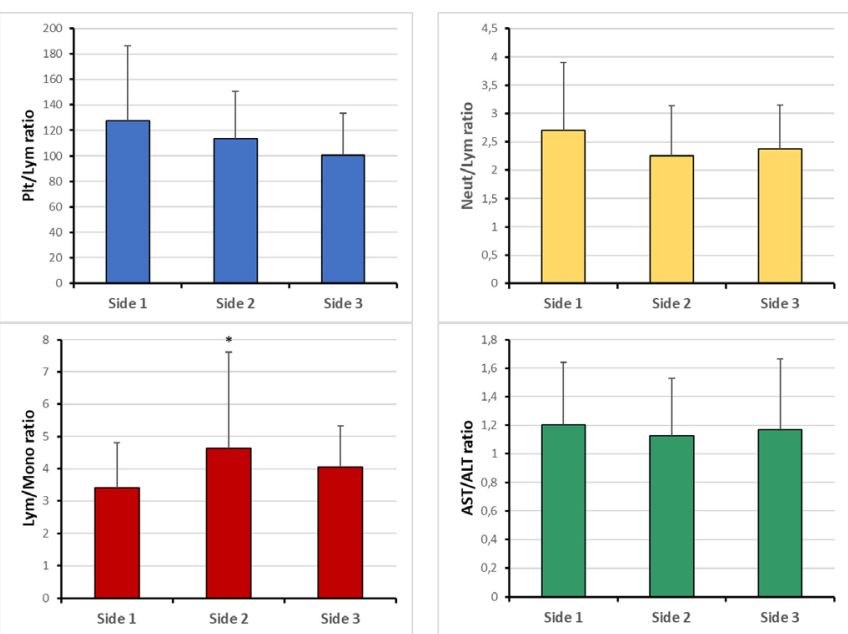

Figure 2. Comparison of Plt/Lym, Neut/Lym, Lym/Mono, and AST/ ALT ratios and side of CAS. Side 1: Left-sided; Side 2: Right-sided; Side 3: Left- and right-sided, ${ }^{*} P<.05$

PCT, Plt/Lym ratio, Lym/Mono ratio, and platelet count. Positive correlations were also detected between MPV and PCT. However, there was a negative correlation between MPV and Lym/Mono ratio. We noted positive correlations between PCT, Lym/Mono ratio, and lymphocyte count. However, negative correlations were observed between Plt/ Lym, Neut/Lym, AST/ALT ratios, and lymphocyte count. There was a positive correlation between monocyte count and PCT. However, there were negative correlations between Plt/Lym, Lym/Mono ratios, and monocyte count. We also observed positive correlations between PCT, Neut/Lym ratio, and neutrophil count (Table 2).

\section{DISCUSSION}

Atherosclerosis is a systemic inflammatory clinical situation that leads to vascular disorder, involving multiple arterial structures. Carotid artery disease is one of the progressive disorders caused by atherosclerosis. Later stage of atherogenesis related to inflammation, endothelial dysfunction, oxidative stress, and smooth muscle cell proliferation play a role in carotid plaque formation [Steinvil 2011; Inaba 2012].

The role of the inflammation at every phase of atherosclerotic course was detected in the literature. $T$ lymphocytes, immunoglobulin molecules, presence of antigen-antibody complex, and plasma cells found in the structure of the atheroma plaque suggest the importance of the inflammation in the development and progression of atherosclerosis, according to the histological studies [Kaplan 2011].

Various inflammatory markers are defined as predictors of inflammation and cardiovascular risk in several studies; these markers include proinflammatory cytokines (interleukin-1, interleukin-6, tumor necrosis factor alpha), oxidized lowdensity lipoproteins, adhesion molecules (intercellular adhesion molecule-1, selectins), acute phase reactants (C-reactive protein, fibrinogen, serum amyloid-A), white blood cells, 
Table 2. Correlations between ratios and biochemical parameters in patients with CAS

\begin{tabular}{|c|c|c|c|}
\hline Platelet count $\leftrightarrow$ PCT & 0.8263 & 0.6828 & $<.0001$ \\
\hline Platelet count $\leftrightarrow$ Neut/Lymratio & -0.1194 & 0.0143 & .2038 \\
\hline Platelet count $\leftrightarrow$ Lym/Mono ratio & 0.3146 & 0.0989 & .0006 \\
\hline $\mathrm{MPV} \leftrightarrow \mathrm{PCT}$ & 0.3706 & 0.1373 & $<.0001$ \\
\hline $\mathrm{MPV} \leftrightarrow \mathrm{Plt} /$ Lymratio & -0.1446 & 0.0209 & .1231 \\
\hline MPV $\leftrightarrow$ Neut/Lymratio & 0.0117 & 0.0001 & .9012 \\
\hline $\mathrm{MPV} \leftrightarrow$ Lym/Mono ratio & -0.3086 & 0.0953 & .0008 \\
\hline $\mathrm{MPV} \leftrightarrow \mathrm{AST} / \mathrm{ALT}$ ratio & -0.0220 & 0.0005 & .8155 \\
\hline Lymphocytes $\leftrightarrow$ Neut/Lymratio & -0.6635 & 0.4403 & $<.0001$ \\
\hline Lymphocytes $\leftrightarrow$ Lym/Mono ratio & 0.4406 & 0.1941 & $<.0001$ \\
\hline Lymphocytes $\leftrightarrow$ AST/ALT ratio & -0.1848 & 0.0342 & .0480 \\
\hline Monocytes $\leftrightarrow$ PCT & 0.2849 & 0.0812 & .0020 \\
\hline Monocytes $\leftrightarrow$ PIt/Lymratio & -0.2520 & 0.0635 & .0066 \\
\hline Monocytes $\leftrightarrow$ Neut/Lymratio & -0.0556 & 0.0031 & .5549 \\
\hline Monocytes $\leftrightarrow$ Lym/Mono ratio & -0.4985 & 0.2485 & $<.0001$ \\
\hline Monocytes $\leftrightarrow \mathrm{AST} / \mathrm{ALT}$ ratio & -0.1337 & 0.0179 & .1542 \\
\hline Neutrophils $\leftrightarrow$ PCT & 0.3287 & 0.1081 & .0003 \\
\hline
\end{tabular}

MPV, mean platelet volume; AST, aspartate aminotransferase; ALT, alanine aminotransferase; PCT, plateletcrit; Neut/Lym, neutrophils/lymphocytes; Plt/Lym, platelets/lymphocytes; Lym/Mono, lymphocytes/monocytes

and erythrocyte sedimentation rate [Pearson 2003]. In addition to these markers, Plt/Lym, Neut/Lym, Lym/Mono, and AST/ALT ratios are novel biomarkers used as indicators of inflammation [Gasparyan 2019; Zhang 2020; Kim 2020; Yayla 2016].

Several previous studies have evaluated the association between white blood cells and their subtypes and presence and prognosis of atherosclerotic process. One of its subtypes, lymphocytes are responsible for humoral and cellular immunity. Inflammation increases the lymphocyte apoptosis and lower lymphocyte counts are correlated with untoward events, in terms of vascular diseases [Gong 2018].

Neut/Lym ratio is an easy, fast, and inexpensive method for assessing inflammatory status and recently has been introduced as a biomarker for investigation of cardiovascular risk.
Ratio of absolute neutrophil count to absolute lymphocyte count is the calculation method of this marker [Forget 2017]. Balta et al investigated the relation between atherosclerosis and Neut/Lym ratio and declared that Neut/Lym ratio can be affected by atherosclerotic risk factors, such as hypertension, diabetes mellitus, hypercholesterolemia, and metabolic syndrome; moreover, it also can be used in the prediction of mortality in cardiovascular diseases [Balta 2016]. Similarly, Kaya et al found correlation between Neut/Lym ratio and severity of coronary artery disease [Kaya 2014]. According to the study designed by Hyun et al in patients with ischemic stroke, it was shown that higher Neut/Lym ratios can be used in prediction of carotid stenosis. Association between Neut/ Lym ratio and carotid artery intima-media thickening was determined in that study [Hyun 2015]. Clinical significance 
of higher Neut/Lym ratio values and internal carotid artery stenosis also was emphasized by Massiot et al [Massiot 2019]. Köklü et al found that the neutrophil count and Neut/Lym ratio were higher in the symptomatic patients than those in the asymptomatic patients with intermediate CAS. They concluded that Neut/Lym ratio can be used as a predictor of stroke risk associated with carotid artery plaques, causing intermediate stenosis [Koklu 2016]. There is evidence that Neut/Lym ratio is increased in the presence of non-calcified carotid artery plaques that cause asymptomatic intermediate CAS [Yuksel 2016]. It has been shown that Neut/Lym ratio was found higher in patients with severe CAS and was positively correlated with the degree of stenosis [Deser 2019]. In our study, Neut/Lym values also were statistically significantly higher in patients with carotid artery stenosis than in the control group $(P<.0001)$, but there was no statistically significant difference in the side of lesion, according to this parameter.

AST/ALT ratio, known as De-Ritis ratio, is an easily applicable, fast and inexpensive blood test that was introduced to evaluate inflammatory status and vascular events. Ratio of absolute AST count to absolute ALT count is the calculation method of this marker [Rief 2016]. Relation between AST/ALT ratio and cardiovascular disease was studied by Yokoyama et al, and it was detected that superior increase in AST/ALT ratio was an independent predictor of all-cause and cardiovascular mortality [Yokoyama 2016]. Elevated AST/ ALT ratio and its association with metabolic syndrome that leads to cardiovascular events were demonstrated by Yadav et al [Yadav 2016]. Similarly, a study designed by Steininger et al showed that increased AST/ALT ratio was associated with a worse outcome after acute myocardial infarction [Steininger 2018]. Hoke et al emphasized that De-Ritis ratio could be used in risk scoring to predict outcomes after carotid artery stenting [Hoke 2012]. In accordance with the literature, AST/ ALT ratio is statistically significantly higher in patients with carotid artery stenosis than the control group in our study $(P<.0001)$, but there was no statistically significant difference in the side of lesion, according to this parameter.

Lym/Mono has been found to be a novel systemic inflammation marker, which is reproducible and widely available in clinical praxis. Ratio of absolute lymphocyte count to absolute monocyte count is the calculation method of this marker. Moreover, a high monocyte count was found to be associated with an increase in carotid intima media thickness that reflects atherosclerotic activity alone [Ganda 2013]. In a study conducted by Gong et al, it was shown that decreased Lym/ Mono ratio were responsible for the adverse outcomes in peripheral arterial occlusive disease [Gong 2018]. Gary et al revealed that decreased Lym/Mono ratio is a risk factor for critical limb ischemia and other vascular peripheral arterial occlusive diseases. The patients who had a transient ischemic attack, due to carotid artery stenosis, had significantly lower Lym/Mono ratios than the control group [Gary 2014]. Similarly, Kurtul et al declared relevance between low Lym/ Mono ratios and no-reflow phenomenon after primary percutaneous coronary intervention for ST-elevated myocardial infarction [Kurtul 2015]. According to Demir et al, lower
Lym/Mono ratios are useful indicators for metabolic syndrome that includes atherosclerosis in vascular arterial structures [Demir 2017]. In a pilot study designed by Switonska et al, it was suggested that lower Lym/Mono ratios are associated with increased severity of stroke [Switonska 2019]. Our study revealed lower but not significant values of Lym/Mono ratios in the carotid artery disease group than the control group $(P<.26)$. In addition, Lym/Mono ratio was found to be statistically significantly higher in left-sided lesions than right-sided lesions, according to our study.

Platelets' role in the initiation of atherosclerotic process has been defined in various studies. Platelets release several mediators, such as platelet activating factor, serotonin, thromboxane A2, platelet derived growth factor and adenosine diphosphate that increase platelet aggregation and activation. They also stimulate chemotaxis for inflammatory cells and fibroblasts and growth of vascular smooth muscle [Thaulow 1991; Nicolsky 2007]. We found positive correlations between PCT, Plt/Lym ratio, Lym/Mono ratio, and platelet count.

$\mathrm{Plt} / \mathrm{Lym}$ ratio is a broadly available and cheap marker that can be used in assessment of inflammation and atherosclerosis. Ratio of absolute platelet count to absolute lymphocyte count is the calculation method of this marker. Its relations with severity and complexity of coronary artery disease in patients with acute coronary syndromes was studied, and increased levels of Plt/Lym ratio was found in association with worse outcomes in those patients [Kurtul 2014]. Akboga et al revealed similar results in patients with stable coronary artery disease. It was declared that $\mathrm{Plt} / \mathrm{Lym}$ ratio was independently and positively associated with the severity of the coronary atherosclerosis [Akboga 2016]. Sari et al mentioned in their study that patients with abnormal coronary angiography results had higher Plt/Lym ratios than patients with normal coronary angiography results. Moreover, it is also correlated with severity of coronary artery disease [Sari 2015]. Similarly, Yuksel et al compared the values of Plt/Lym ratio between the patients with severe atherosclerosis, mild atherosclerosis, and control groups and revealed that mean $\mathrm{Plt} / \mathrm{Lym}$ ratio of the severe atherosclerotic group was significantly higher than those two [Yuksel 2016]. Massiot et al mentioned about the relationship between the high PLR and symptomatic internal carotid artery severity [Massiot 2019]. Bonaventura et al suggested that PLR can be used in prediction of acute coronary syndrome development at 18 -month follow up after carotid endarterectomy patients with severe carotid artery atherosclerosis [Bonaventura 2020]. Soylu et al concluded that increased platelet-to-lymphocyte ratio could be a marker of the clinical course in patients with carotid arterial stenosis [Soylu 2017]. According to our study, PLR is higher in the group of patients with carotid artery disease than the control group, but there was no statistically significant difference in the side of lesion according to this parameter. We also found positive (platelet count) and negative (lymphocyte, monocyte counts) correlations with Plt/ Lym ratio. 
CONCLUSION

Plt/Lym, Neut/Lym, AST/ALT, and Lym/Mono ratios are fast, easy, inexpensive, and reproducible parameters that can be used in determining the prediction of carotid artery stenosis. We reached this conclusion using a retrospective study. Larger prospective and multicenter studies must be performed to explain the relationship between the CAS and these parameters.

\section{REFERENCES}

Akboga MK, Canpolat U, Yayla C, Ozcan F, Ozcan O, Topaloglu S, et al. 2016. Association of platelet-to-lymphocyte ratio with inflammation and severity of coronary atherosclerosis in patients with stable coronary artery disease. Angiology 67(1):89-95.

Balta S, Celik T, Mikhailidis DP, Ozturk C, Demirkol S, Aparci M, et al. 2016. The relation between atherosclerosis and the neutrophil-lymphocyte ratio. Clin Appl Thromb Hemost 22(5):405-411.

Bonaventura A, Carbone F, Liberale L, Mach F, Roth A, Burger F, et al. 2020. Platelet-to-lymphocyte ratio at the time of carotid endarterectomy is associated with acute coronary syndrome occurrence. J Cardiovasc Med 21(1):80-82.

Brott TG, Halperin JL, Abbara S, Bacharach JM, Barr JD, Bush RL, et al. 2011. 2011 ASA/ACCF/AHA/AANN/AANS/ACR/ASNR/CNS/SAIP/ SCA guideline on the management of patients with extracranial carotid and vertebral artery disease. J Am Coll Cardiol 57(4):16-94.

Chrysohoou C, Pitsavos C, Panagiotakos DB, Skoumas J, Stefanidis C. 2004. Association between prehypertension status and inflammatory markers related to atherosclerotic disease: The ATTICA Study. AJH 17(2):568-573.

Demir V, Akboga MK, Yilmaz S, Ede H. 2017. Assessment of monocyte-to-high density lipoprotein cholesterol ratio and lymphocyte-tomonocyte ratio in patients with metabolic syndrome. Biomark Med 11(7):535-540.

Deser SB, Yucel SM, Demirag MK, Guclu MM, Kolbakir F, Keceligil HT. 2019. The association between platelet/lymphocyte ratio, neutrophil/lymphocyte ratio, and carotid artery stenosis and stroke following carotid endarterectomy. Vascular 27(6):604-611.

Forget P, Kahfia C, Defour JP, Latinne D, VanPel MC, De Kock M. 2017. What is the normal value of the neutrophil-to-lymphocyte ratio? BMC Research Notes 10(12):233-238.

Ganda A, Magnusson M, Yvan Charvet L, Hedbald B, Engström G, Eller P, et al. 2013. Mild renal dysfunction and metabolites tied to low HDL cholesterol are associated with monocytosis and atherosclerosis. Circulation 127(3):988- 996.

Gary T, Pichler M, Belaj K, Eller P, Hafner F, Gerger A, et al. 2014. Lymphocyte-to-monocyte ratio: a novel marker for critical limb ischemia in PAOD patients. Int J Clin Pract 68(3):1483-1487.

Gasparyan AY, Ayvazyan L, Mukanova U, Yessirkepov M, Kitas GD. 2019. The platelet-to-lymphocyte ratio as an inflammatory marker in rheumatic diseases. Ann Lab Med 39(4):345-357.

Gong S, Gao X, Xu F, Shang Z, Li S, Chen W, et al. 2018. Association of lymphocyte-to-monocyte ratio with severity of coronary artery disease. Med (Baltimore) 97(43):e12813.

Hoke M, Ljubuncic F, Steinwender C, Huber K, Minar E, Koppensteiner
R, et al. 2012. A validated risk score to predict outcomes after carotid stenting. Circulation 5(6):841-849.

Hyun S, Kwon S, Cho SY, Park S, Jung WS, Moon SK, et al. 2015. Can the neutrophil-to-lymphocyte ratio appropriately predict carotid artery stenosis in patients with ischemic stroke?- A retrospective study. J Strok Cerebrovasc Dis 24(11):2646-2651.

Inaba Y, Chen JA, Bergman SR. 2012. Carotid plaque, compared with carotid intima-media thickness, more accurately predicts coronary artery disease events: A meta-analysis. Atherosclerosis 220(1):128-133.

Kaplan ZS, Jackson SP. 2011. The role of platelets in atherothrombosis. Hematology Am Soc Hematol Educ Program 2011(2):51-61.

Kaya H, Ertas H, Islamoglu Y, Kaya Z, Atilgan ZA, Cil H, et al. 2014. Association between neutrophil to lymphcyte ratio and severity of coronary artery disease. Clin Appl Thromb Hemost 20(1):50-54.

Kim HJ, Kim SY, Shin SP, Yang YJ, Bang CS, Baik GH, et al. 2020. Immunological measurement of aspartate/alanine aminotransferase in predicting liver fibrosis and inflammation. Korean J Intern Med 35(2):320-330.

Koklu E, Yuksel IO, Arslan S, Bayar N, Cagırcı G, Gencer ES, et al. 2016. Is elevated neutrophil-to-lymphocyte ratio a predictor of stroke in patients with intermediate carotid artery stenosis? J Stroke Cerebrovasc Dis 25(3):578-84.

Kristensen SD. 1992. The platelet-vessel wall interaction in experimental atherosclerosis and ischaemic heart disease with special reference to thrombopoiesis. Dan Med Bull 39(3):110-127.

Kurtul A, Sani NM, Yarlioglues M, Duran M, Ergun G, Acikgoz SK, et al. 2014. Association of platelet-to-lymphocyte ratio with severity and complexity of coronary artery disease in patients with acute coronary syndrome. Am J Cardiol 114(7):972-978.

Kurtul A, Yarlioglues M, Celik IE, Duran M, Elcik D, Kilic A, et al. 2015. Association of lymphocyte-to-monocyte ratio with the no-reflow phenomenon in patients who underwent a primary percutaneous coronary intervention for ST-elevation myocardial infarction. Coron Artery Dis 26:706-712.

Massiot N, Lareyre F, Pons AV, Pelletier Y, Chikande J, Carboni J, et al. 2019. High neutrophil to lymphocyte ratio and platelet to lymphocyte ratio are associated with symptomatic internal carotid artery stenosis. J Strok Cerebrovasc Dis 28(1):76-83.

Nicolsky E, Grines CL, Cox DA, Garcia E, Tcheng JE, Sadeghi M, et al. 2007. Impact of baseline platelet count in patients undergoing primary percutaneous coronary intervention in acute myocardial infarction (from the CADILLAC trial). Am J Cardiol 99(1):1055-1061.

Pearson TA, Mensah GA, Alexander RW, Anderson JL, O Cannon R, Criqui M, et al. 2003. Markers of inflammation and cardiovascular disease: application to clinical and public health practice: A statement for healthcare professionals from the Centers for Disease Control and Prevention and the American Heart Association. Circulation 107(3):499-511.

Rief P, Pichler M, Raggam R, Hafner F, Gerger A, Eller P, et al. 2016. The AST/ALT (De-Ritis) ratio: A novel marker for critical limb ischemia in peripheral arterial occlusive disease patients. Medicine (Baltimore) 95(24):e5074.

Sari I, Sunbul M, Mammadov C, Durmus E, Bozbay M, Kivrak T, et al. 2015. Relation of neutrophil-to-lymphocyte and platelet-to-lymphocyte ratio with coronary artery disease severity in patients undergoing coronary angiography. Kardiol Pol 73(12):1310-1316. 
Soylu AI, Cortcu SA, Uzunkaya F, Atalay YO, Bekci T, Gungor L, et al. 2017. The correlation of the platelet-to-lymphocyte ratio with the severity of stenosis and stroke in patients with carotid arterial disease. Vascular 25(3):299-306.

Steininger M, Winter MP, Reiberger T, Koller L, El-Hamid F, Forster S, et al. 2018. De-Ritis ratio improves long-term risk prediction after acute myocardial infarction. J Clin Med 7(12):474-483.

Steinvil A, Sadeh B, Arbel Y, Justo D, Belei A, Borenstein N, et al. 2011. Prevalence and predictors of concomitant carotid and coronary artery atherosclerotic disease. J Am Coll Cardiol 57(7):779-783.

Switonska M, Slomka A, Korbal P, Slomka NP, Sinkiewicz W, Sokal P, et al. 2019. Assessment of neutrophil-to-lymphocyte ratio and lymphocyteto-monocyte ratio with treatment modalities of acute ischaemic stroke: A pilot study. Medicina 55(7):342-349.

Thaulow E, Erikssen J, Sandvik L, Stormorken H, Cohn PF. 1991. Blood platelet count and function are related to total and cardiovascular death in apparently healthy men. Circulation 84(1):613-617.

Tsiara S, Elisaf M, Jagroop IA, Mikhailidis DP. 2003. Platelets as predictor of vascular risk: is there a practical index of platelet activity? Clin Appl Thromb Hemost 9:177-190.

Varım C, Varım P, Acar BA, Vatan MB, Uyanik MS, Kaya T, et al. 2016. Usefulness of the platelet-to-lymphocyte ratio in predicting the severity of carotid artery stenosis in patients undergoing carotid angiography.
Kaohsiung J of Med Sci 32(2):86-90.

Yadav D, Choi E, Ahn SV, Baih SK, Cho YZ, Koh SB, et al. 2016. Incremental predictive value of serum AST-to-ALT ratio for incident metabolic syndrome: The ARIRANG Study. PloS One 11(8):e0161304.

Yayla C, Akboga MK, Yayla KG, Ertem AG, Efe TH, Sen F, et al. 2016. A novel marker of inflammation in patients with slow coronary flow: lymphocyte-to-monocyte ratio. Biomark Med 10(5):485-493.

Yokoyama M, Watanebe T, Otaki Y, Takahashi H, Arimoto T, Shishido T, et al. 2016. Association of the aspartate aminotransferase to alanine aminotransferase ratio with BNP level and cardiovascular mortality in the general population: The Yamagata Study 10-year follow up. Dis Markers 2016(1):1-9.

Yuksel IO, Koklu E, Arslan S, Cagırcı G, Goksu EO, Koc P, et al. 2016. Association of neutrophil/lymphocyte ratio with plaque morphology in patients with asymptomatic intermediate carotid artery stenosis. Korean Circ J 46(5):699-705.

Yuksel M, Yildiz A, Oylumlu M, Akyuz A, Aydin M, Kaya H, et al. 2016. The association between platelet/lymphocyte ratio and coronary artery disease severity. Anatol J Cardiol 15(8):640-647.

Zhang WB, Zeng YY, Wang F, Cheng L, Tang WS, Wang WQ. 2020. A high neutrophil-to-lymphocyte ratio predicts hemorrhagic transformation of large atherosclerotic infarction in patients with acute ischemic stroke. Aging 12(3):2428-2439. 